\title{
Electro-mechanical Behavior of Graphene-Polystyrene Composites Under Dynamic Loading
}

\author{
N. Heeder • I. Chakraborty $\cdot$ A. Bose • \\ A. Shukla
}

Received: 26 December 2014/ Accepted: 16 January 2015/Published online: 6 February 2015

(C) Society for Experimental Mechanics, Inc 2015

\begin{abstract}
An experimental investigation was conducted to understand the electro-mechanical response of graphene reinforced polystyrene (PS) composites under static and dynamic loading. Graphene/PS composites were fabricated using a solution mixing approach followed by hot-pressing. Absolute resistance values were measured with a highresolution four-point probe method for both quasi-static and dynamic loading. A modified split Hopkinson (Kolsky) pressure bar apparatus, capable of simultaneous mechanical and electrical characterization, was developed and implemented to investigate the dynamic electro-mechanical response of the composites. In addition to measuring the change in electrical resistance as well as the dynamic constitutive behavior, real-time surface damage and global deformation was captured using high-speed photography. The real-time damage was correlated to both stress-strain and percent change in resistance profiles. The experimental findings indicate that the bulk resistance of the composite increased significantly due to the brittle nature of the PS matrix and the presence of relative agglomerations of graphene platelets which resulted in micro-
\end{abstract}

\footnotetext{
N. Heeder · A. Shukla $(\bowtie)$

Dynamic Photo Mechanics Laboratory, Department of Mechanical, Industrial \& Systems Engineering, University of Rhode Island, Kingston, RI 02881, USA

e-mail: shuklaa@egr.uri.edu

N. Heeder

e-mail: nicholas.heeder@gmail.com

I. Chakraborty · A. Bose

Department of Chemical Engineering, University of Rhode Island, Kingston, RI 02881, USA

e-mail: chakrabortyi@egr.uri.edu

A. Bose

e-mail: bosea@egr.uri.edu
}

crack formations. Scanning electron microscopy imaging gives further insight into the various damage mechanisms that occur within the composites subjected to a static or dynamic load. The results show that the change in transport properties can provide further insight into the microstructural evolution of composite materials during loading.

Keywords Electrical response - Graphene/polymer composites · Dynamic response - Quasi-static response . Four-point probe method

\section{Introduction}

A comprehensive series of experiments were conducted to experimentally investigate the electro-mechanical response of graphene/polystyrene (PS) composites subjected to static and dynamic split Hopkinson pressure bar (SHPB) loading. A novel SHPB apparatus, capable of simultaneous mechanical and electrical characterization, was developed to effectively investigate the electro-mechanical response of the graphene reinforced PS composites. The history between the electrical resistance change, mechanical loading, and the high-speed deformation photography are correlated to characterize the electrical-mechanical response of the fabricated composites. Furthermore, scanning electron microscopy (SEM) imaging was performed to give further insight into the various damage mechanisms that occur within the composites when a static or dynamic load is applied.

Owing to extraordinary physical and mechanical properties, graphene has the potential to be an ideal filler material in developing novel composites with multifunctional capabilities such as self-sensing and active response. Recent progress has shown that inorganic nanomaterials as 
fillers in polymer/inorganic composites have tremendous application potential in industries such as automotive, aerospace, construction and electronics [1-11]. Although carbon nanotubes (CNTs) possess comparable mechanical properties, graphene still has superior electrical and thermal properties, and a higher surface area [4, 12]. Its reinforcement can offer exceptional properties in future high performance novel composites. In recent years, graphene based composites have become a topic of significant academic and industrial interest. While a number of studies have shown that the presence of graphene within polymers can enhance the mechanical properties of the bulk composite [12-18], other studies have shown that graphene can also have adverse effects on the mechanical properties [1921]. Fang et al. [13] investigated the effect of low concentrations of graphene on the mechanical strength of graphene/PS composites. The results showed a substantial increase in tensile strength as graphene loadings were increased from 0.1 to $0.9 \mathrm{wt} \%$ in comparison to pristine PS. The increase in strength was attributed to effective load transfer between the graphene and polymer. By contrast, the addition of certain filler materials can also have adverse effects on the mechanical properties of the resulting composite due to factors such as reinforcement phase concentration, dispersion quality, interface bonding, aspect ratio, surface-to-volume ratio of filler, etc. [12, 19-24]. Wang et al. [24] compared the use of graphite nanosheets to carbon black as a filler material in high density polyethylene. They reported a gentle increase in both tensile strength and impact strength of the composite with low loadings of graphene $(0.5-2 \mathrm{wt} \%)$ but a sharp reduction when the graphene content was greater than $2 \mathrm{wt} \%$. Due to the high surface energy of graphene, as well as the weak interaction between the graphene and polyethylene, an inhomogeneous dispersion in the polymer matrix was formed when the content of graphene was high, leading to adverse effects on the properties of the composites.

Due to the exceptional electrical properties of graphene, several researchers in the past have also studied the utilization of graphene as an electrically conductive additive in composites $[3,23,25,26]$. The electrical conductivity of graphene-based composites has been studied theoretically [27] and as experimentally [23, 26, 28, 29]. Studies have shown remarkable increases in composite electrical properties with graphene reinforcement. More recently, Qi et al. [26] demonstrated a substantial enhancement of electrical properties of PS with the addition of graphene. The conductivity of the graphene/PS composite was shown to be $\sim 2-4$ orders of magnitude higher than that of multi-walled-carbon-nanotube/PS composites. The combination of the remarkable mechanical properties and the exceptional electrical properties makes graphene an ideal candidate for use as a filler material in fabricating multi-functional composites capable of sensing material behavior. Many reports demonstrate the effectiveness of utilizing CNT reinforced polymer composites as strain and damage sensors [30-33]. More recently, similar studies have been conducted where graphene is utilized to provide strain sensing functions [34-36]. Eswaraiah et al. [34] demonstrated the real time strain response of $\mathrm{f}-\mathrm{G}-\mathrm{PVDF}$ composites on the macro-scale under tensile loads and the use of the composite as a strain sensor. Analysis of the change in voltage of various composite films revealed that the graphenebased composite had better strain sensing performance than carbon nanotube-based polymer composites.

The aforementioned studies revealed that when properly dispersed within a given matrix, an internal sensory network can be formed and utilized to detect important information such as strain and damage within the material. To further this investigation, it is crucial to understand the electrical response of graphene reinforced composites under dynamic compressive loading conditions. The present study experimentally investigates the electro-mechanical response of graphene/PS composites subjected to static and dynamic SHPB loading. A novel SHPB apparatus, capable of simultaneous mechanical and electrical characterization, was developed to effectively investigate the electro-mechanical response of the graphene reinforced PS composites. The history between the electrical resistance change, mechanical loading, and the high-speed deformation photography are correlated to characterize the electrical-mechanical response of the fabricated composites. To give further insight, scanning electron microscopy (SEM) imaging was used to show various damage mechanisms that occur within the composites when a static or dynamic load is applied.

\section{Material and Specimen}

\section{Material Fabrication}

The graphene nanoplatelets (GNPs) used in this study were $\mathrm{xGnP}^{\mathrm{TM}}$ Nanoplatelets (XG Sciences). These nanoparticles consist of short stacks of one or more graphene sheets having a lateral dimension of $\sim 25 \mu \mathrm{m}$ and a thickness of $\sim 6 \mathrm{~nm}$. This thickness corresponds to approximately 18 graphene layers at a typical graphite interlayer spacing. It has been proposed that materials of this thickness $(>10$ layers) be referred to as exfoliated graphite, or graphite nanoplatelets for scientific classification [37]. The same materials are sometimes marketed by suppliers as "graphene nanoplatelets". An SEM image of these platelets is shown in Fig. 1. The specific polymeric matrix chosen for this study was PS (Crystal PS 1300) purchased from Styrolution. The PS had an average molecular weight of about $121,000 \mathrm{~g} / \mathrm{mol}$. 


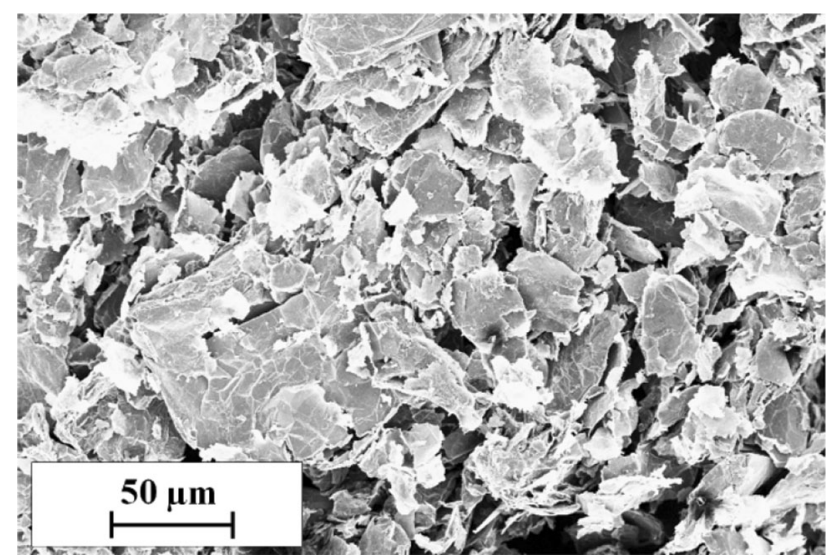

Fig. 1 SEM image of xGnP M-25 nanoplatelets

Graphene's strong intrinsic van der Waals forces of attraction between sheets and high surface area make graphene very difficult to disperse uniformly within polymer materials [24]. In order to disperse the platelets throughout the PS matrix, a solution mixing process was employed [26]. The general procedure used to disperse the graphene platelets is shown in Fig. 2. First, $5 \mathrm{~g}$ of PS was dissolved in $30 \mathrm{~mL}$ of dimethyl formamide (DMF). The desired amount of GNPs was dispersed in a separate DMF solution $(\sim 0.1 \mathrm{~g}$ graphene per $100 \mathrm{~mL}$ DMF) using ultrasonication. The GNP/DMF solution was sonicated for $1.5 \mathrm{~h}$ at $20 \mathrm{kHz}$ on pulse mode, 30 on 10 s off using a Sonics \& Materials Inc. VCX750 probe sonicator. The GNP/DMF suspension was then added to the PS/DMF solution and mechanically stirred for $\sim 2 \mathrm{~h}$. Since the nanoplatelets tend to agglomerate during slow solvent evaporation, the solution was dropped into a large volume of methanol to coagulate the GNP/PS composites. The resulting composite was then filtered and dried in an oven at $\sim 80{ }^{\circ} \mathrm{C}$ for $\sim 18 \mathrm{~h}$. Finally, the dried GNP/PS composites were hot-pressed using a heated steel mold $\left(\sim 190{ }^{\circ} \mathrm{C}\right)$ and a hydraulic press.

\section{Specimen}

Figure 3 illustrates specimens prepared for both quasistatic and dynamic compression loading experiments. To provide sufficient electrical conductivity, 5 vol\% GNP/PS composites were fabricated for all experiments. Specimens used in quasi-static experiments were $10 \mathrm{~mm}$ in length and had a diameter of $6.35 \mathrm{~mm}$, where the loading was exerted in the longitudinal direction. Specimens used in dynamic experiments were $8.68 \mathrm{~mm}$ in length and had a diameter of $15.87 \mathrm{~mm}$. Two V-notch channels with a depth of $0.3 \mathrm{~mm}$ were machined in the middle section of both specimens located $1.9 \mathrm{~mm}$ from each face. The channels were used to implement a modified four-point probe method [33] in order to effectively measure the change in electrical resistance of the specimen during loading. The loading was exerted in the longitudinal direction. The left face, right face, and the two inner channels of the specimen served as four probes to obtain a four point probe measurement. All four probes were coated with silver paint (SPI-Paint 05001-AB) and lead wires were attached using an adhesive (M-Coat A Air-Drying Polyurethane Coating).

\section{Experimental Setup and Procedure}

\section{Electrical Characterization}

In order to effectively capture the change in electrical resistance of the cylindrical specimen, a novel approach
Fig. 2 Procedure for dispersing GNPs in polystyrene

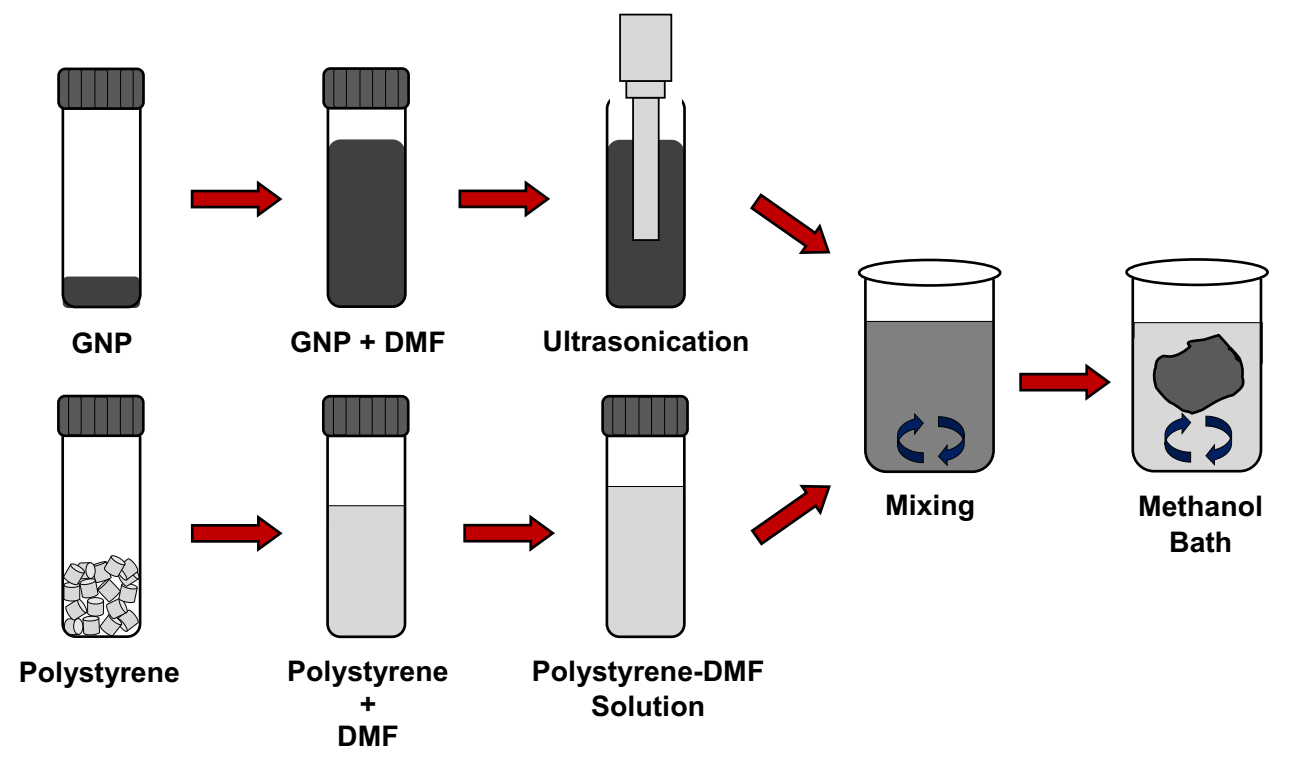




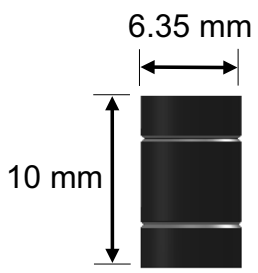

(a)

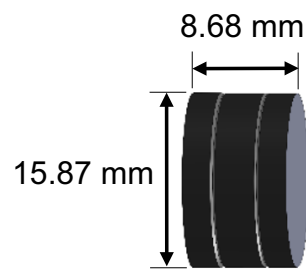

(b)
Fig. 3 Specimen geometry used for a quasi-static loading and b dynamic loading

previously developed by the authors utilizing the fourpoint probe method was implemented [31]. The four probes consisted of the left face, right face, and the two inner channels. To allow a constant current flow through the entire bulk of the specimen, a constant current was supplied through the right and left faces of the specimen. The two inner channels served as the two peripheral electrodes that measure the voltage drop across the middle section of the specimen. The electrical resistance of the middle section can be easily determined from the input current and the voltage drop across the inner probes. As the specimen underwent deformation, the instantaneous resistance of the middle section changed. Percent change in the resistance was calculated for each experiment. Due to the complex dispersion pattern of graphene inside, the initial resistance of the individual specimens varies slightly. Therefore, the initial resistance of each specimen served as the baseline for each experiment. Based on previous studies [31], this method better provides the means to detect changes in the resistance caused by strain and damage mechanisms in the material as compared to the classical four-point probe method. Since the current flows through the cross sectional area, the measured resistance is an estimation of the bulk resistance of the inner section. By using this average voltage measurement technique, more consistent and accurate results were obtained during a wide range of mechanical loading schemes and consequent specimen deformations.

\section{Quasi-Static Electro-mechanical Characterization}

The quasi-static loading was implemented by a screw-driven testing machine. A modified four-point probe method was utilized to measure the resistance change during the compression tests [31]. The experimental setup used to capture the resistance change of the composites under quasi-static loading is shown in Fig. 4. A constant current source was used to supply a DC current flow through the specimen. The GNP/PS specimen was sandwiched between two aluminum plates to establish uniform current flow through the specimen during the compressive loading. Silver paint was applied to the top and bottom of each specimen to minimize the contact resistance between the specimen and the plates. Each loading head was insulated from the electrical measurement system. Two electrometers were used to measure the voltage at each of the two inner probe rings. The difference between the two voltage readings, which corresponds to the voltage drop across the two inner probes, was measured using a digital multimeter and recorded using a LabView system.

\section{Dynamic Electro-mechanical Characterization}

A modified split Hopkinson (Kolsky) pressure bar apparatus, capable of simultaneous mechanical and electrical characterization, was developed and implemented to investigate the dynamic electro-mechanical response of the GNP/PS composites.

A typical SHPB consists of a striker bar, a solid incident bar and a solid transmission bar. The striker bar is propelled using an air-operated gun. A pulse shaper is commonly placed at the impact end of the incident bar with a thin layer of lubricant to improve force equilibrium conditions at the specimen-bar interfaces. The theoretical details of SHPB can be obtained from Kolsky [38]. The specimen is sandwiched between the incident bar and the transmission bar. A lubricant is applied between the specimen and the bar interfaces to minimize friction.

When the striker bar impacts the incident bar, an elastic compressive stress pulse, referred to as the incident pulse,
Fig. 4 Experimental setup for electrical characterization under quasi-static loading

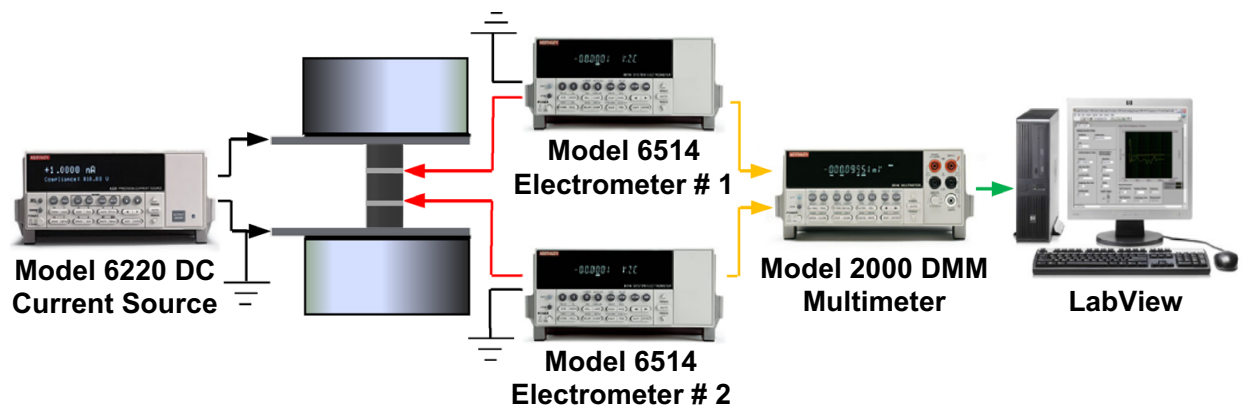


Fig. 5 Experimental setup of SHPB apparatus with dynamic electrical characterization setup

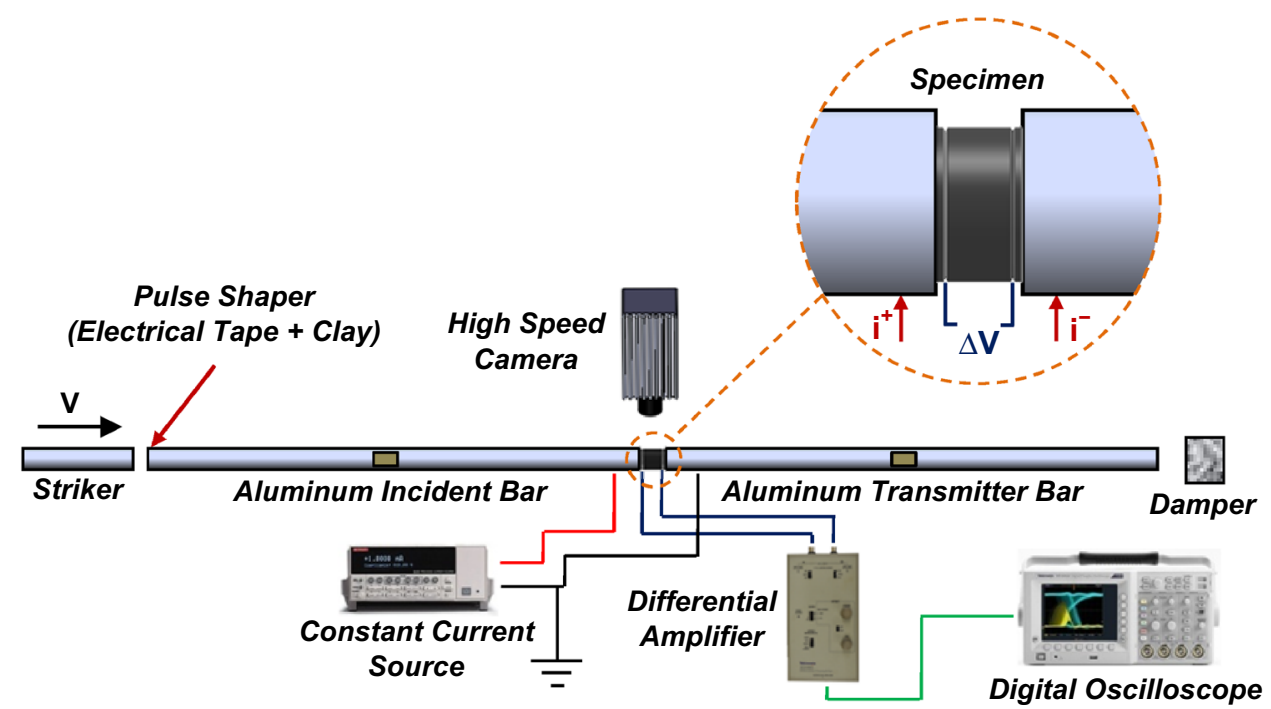

is generated and then propagates along the incident bar towards the specimen. When the incident pulse reaches the specimen, part of it reflects back into the incident bar (reflected pulse) in the form of a tensile pulse due to the impedance mismatch at the bar-specimen interface, and the remaining pulse is transmitted (transmission pulse) to the transmission bar. Axial strain gages mounted on the surfaces of the incident and transmission bars provide timeresolved measures of the elastic strain pulses in the bars. The length of the incident pulse is related to the projectile length which allows for variation in achievable strain rates.

Using one-dimensional wave theory, the engineering stress and engineering strain in the specimen can be determined from the reflected and transmitted strain pulses respectively, as given in Eqs. 1 and 2.

$\sigma_{s}=E_{b} \frac{A_{b}}{A_{s}} \varepsilon_{t}(t)$

$\varepsilon_{s}=\frac{-2 c_{b}}{L_{s}} \int_{0}^{t} \varepsilon_{r}(t) d t$

The above equations were suitably modified to obtain the true stress and true strain in the specimen. The expressions for the forces at the specimen incident bar interface and at the specimen transmission bar interface are given in Eqs. 3 and 4 respectively.

$F_{i}=A_{b} E_{b}\left(\varepsilon_{i}+\varepsilon_{r}\right)$

$F_{t}=A_{b} E_{b} \varepsilon_{t}$

where $\varepsilon_{i}, \varepsilon_{r}, \varepsilon_{t}$ are the time-resolved strain values of the incident, reflected and transmitted pulses respectively, $c_{b}=\sqrt{E_{b} / \rho_{b}}$ is the longitudinal bar wave speed, $E_{b}$ is the Young's modulus of the bar material, $\rho_{b}$ is the density of the bar material, $L_{s}$ is the thickness of the specimen, $A_{b}$ is the cross-sectional area of the bar and $A_{s}$ is the cross-sectional area of the specimen. Force equilibrium within the specimen during the wave loading is attained when the forces on each face of the specimen are equal.

Several modifications were made to the existing SHPB to simultaneously capture the electrical response as well as the mechanical behavior of the specimen during the dynamic loading. A sketch of the novel SHPB device is shown in Fig. 5. The aluminum incident and transmission bars were $19.05 \mathrm{~mm}$ in diameter and measured 1,613 and 1,220 $\mathrm{mm}$ in length respectively. Lead wires were securely attached to each bar to provide a means of supplying a DC current flow through the specimen during loading. In order to obtain an accurate electrical response of the specimen, nylon bushings were fabricated and installed to isolate the incident and transmission bars from the supports. A similar four-point probe technique, as described in quasi-static experiments, was implemented. To minimize the contact resistance and the frictional forces present at the specimenbar interfaces, a conductive lubricant (AI Technology Inc. ELGR8501) was applied to the specimen faces. Additionally, a pulse shaper consisting of a single layer of electrical tape and clay ( $\sim 2 \mathrm{~mm}$ thick) were used to isolate the incident bar from the gas gun apparatus and to improve the force equilibrium conditions at the specimen-bar interfaces. A constant current source with high frequency response (Keithley Instruments Model 6221) was used to supply the constant DC current flow under the high rate deformation while the voltage drop between the two inner probes was measured by a differential amplifier (Tektonix ADA 400A) and recorded by a digital oscilloscope (Tektronix TDS 3014). 


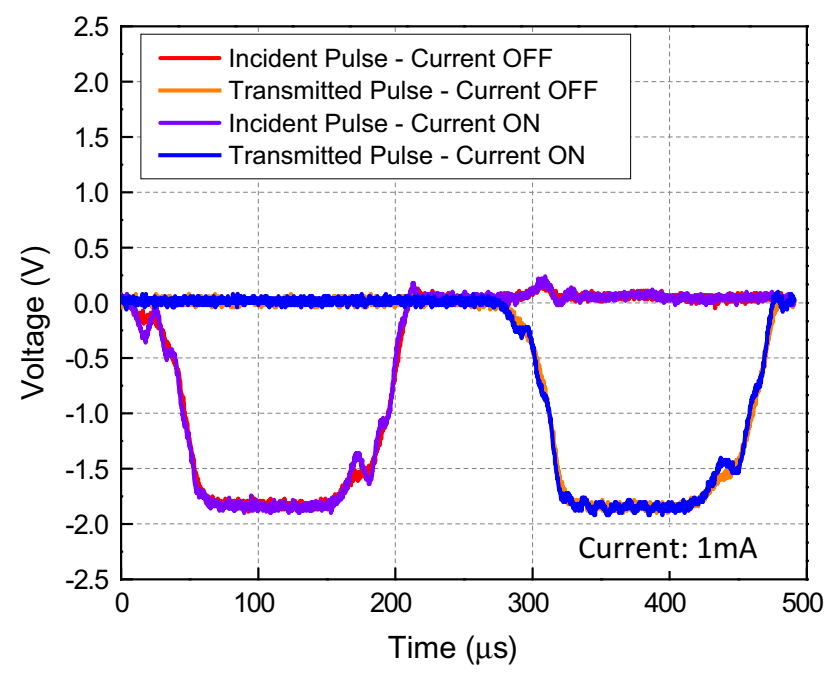

Fig. 6 Original pulses generated from the modified SHPB to ensure no current effect

It is important to note that proper strain gage selection is critical in preventing any electrical interference in strain measurements while conducting these types of experiments. The particular strain gages chosen (MicroMeasurements C2A-13-250LW-350) consisted of an encapsulated gage mounted on a thin high-performance laminated polyimide film backing. The polyimide film backing provides a layer of insulation between the actual gage and the bar surface and therefore prevents any voltage interference. A series of experiments were performed with and without supplying current through the bars, validating that the strain gages bonded to the bars remain unaffected. Figure 6 shows typical pulses obtained from the strain gages for the two cases when the incident bar is in contact with the transmission bar without any specimen in between. Since there is no impedance mismatch at the bars interface, the entire incident pulse is transmitted to the transmission bar. It can be clearly seen that there is no effect on the pulses when a current of $1 \mathrm{~mA}$ was supplied through the bars.

In order to fully characterize the change in transport properties of the GNP/PS composites under high strain rates, two different series of experiments were conducted. The first series of dynamic experiments comprised of loading the composites until complete dynamic failure. Once the complete electro-mechanical response of the composites was understood, a series of controlled-strain experiments were designed and carried out to provide more insight on the internal damage mechanisms occurring during the dynamic event.

In the first series of dynamic experiments, where the composite was loaded to complete failure, a striker length of $406 \mathrm{~mm}$ was used to achieve large strains in the specimen. The second series of dynamic experiments, where the composite was loaded to a strain below the failure strain, required additional modification to the electro-mechanical SHPB apparatus. To ensure that a single compressive loading was applied to the specimen and to control the amount of strain the specimen undergoes, a momentum trap was built and installed on the SHPB. The momentum trap consisted of a flange, mounted on the impact end of the incident bar, and a rigid mass [39]. A striker length of $100 \mathrm{~mm}$ was used for all the controlled-strain experiments, and the gun pressure was varied to ensure the desired incident pulse profile. A high-speed digital camera (Photron SA1) was used to capture the real time deformation of the specimen at a frame rate of $100,000 \mathrm{fps}$.

\section{Experimental Results and Discussion}

\section{Quasi-Static Compressive Response}

A typical electro-mechanical response of a 5 vol\% GNP/PS composite under compressive loading is shown in Fig. 7. During the quasi-static compression, the stress on the specimen monotonically increases to $47 \mathrm{MPa}$ at $5 \%$ strain and then gradually decreases. Taking the initial resistance $R_{o}$ as a baseline, the percent change in electrical resistance increases proportionally with strain. Initially, no significant change in resistance is observed up until $\sim 1 \%$ strain. Due to the brittle nature of the PS matrix, small micro-cracks begin to form as the compressive strain increases resulting in a substantial increase in electrical resistance. Since the electrical resistance of the matrix material is very high, the graphene particles exclusively conduct the electrical current within the material. When considering the negligible change in graphene particle geometry during the compressive event, it is evident that the resistance change is

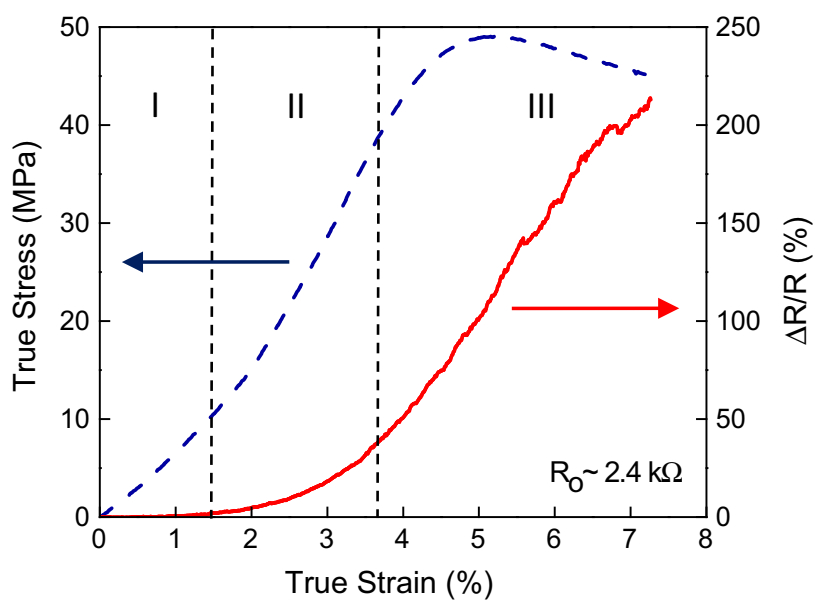

Fig. 7 Typical electro-mechanical response of 5 vol\% GNP/PS under quasi-static loading 
caused by the interruptions of the electrical networks between the graphene particles.

A schematic representing the damage mechanisms induced by the mechanical compression is shown in Fig. 8 . As observed in Fig. 8I, the electrical resistance of the composite begins to increase at $\sim 1 \%$ strain due to the disruption of the graphene network present throughout the volume of the composite. Scanning electron microscopy (SEM) was performed on the post-mortem specimens to provide more insight on the internal damage mechanisms occurring during the compressive event. As shown in Fig. 9, there is evidence of micro-cracks located primarily around small agglomerates of GNP. The small agglomerates of the graphene sheets appear to serve as crack nucleation sites and due to poor interactions between the PS matrix and GNPs, damage tends to propagate along the graphene-polymer interfaces. The micro-cracks, formed primarily around the graphene agglomerations, continue to grow and propagate throughout the composite which causes the rate of electrical resistance to increase $\sim 6.5$ times. The formation of additional voids and cracks due to the increasing strain further decreases the electrical efficiency between graphene particles, which is illustrated in Fig. 8II and III.

The true stress versus true strain curves for pristine PS and 5 vol\% GNP/PS are shown in Fig. 10. A significant decrease in yield strength $(\sim 47 \%)$ and modulus $(\sim 57 \%)$ is observed with the addition of graphene. This result differs from most reports where authors observed significant

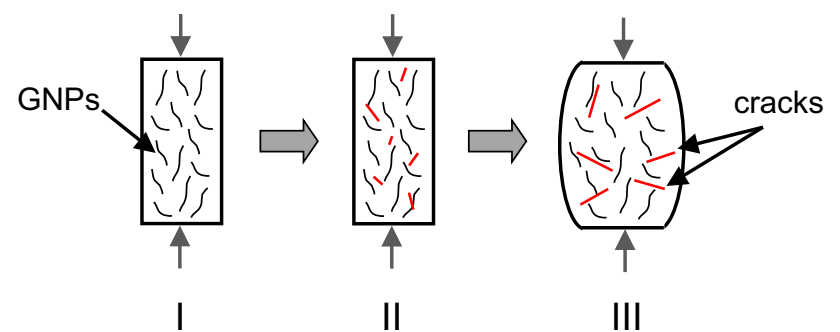

Fig. 8 Representation of a cross-section of a GNP/PS composite under compressive loading improvements in mechanical properties when graphene was used as a filler material within various polymers [12, $13,15,16]$. Generally, the enhancement of strength and modulus is attributed to high aspect ratio and high strength of the filler as well as the uniform distribution and good interfacial adhesion between the fillers and matrixes, which provide effective load transfer from the matrixes to the fillers $[12,24,40]$. When relatively high concentrations of graphene are used, an ineffective dispersion typically forms due to inevitable aggregation of the graphene particles. The heterogeneity of the polymer microstructure creates many structural flaws and weak interfaces between the GNPs and PS resulting in a decrease in mechanical strength. This behavior has been previously reported and demonstrated for various types of particles, including graphene [12, 1924].

Split Hopkinson Pressure Bar Loading

To investigate the electrical response of the graphene reinforced composites under higher strain rates, the electro-

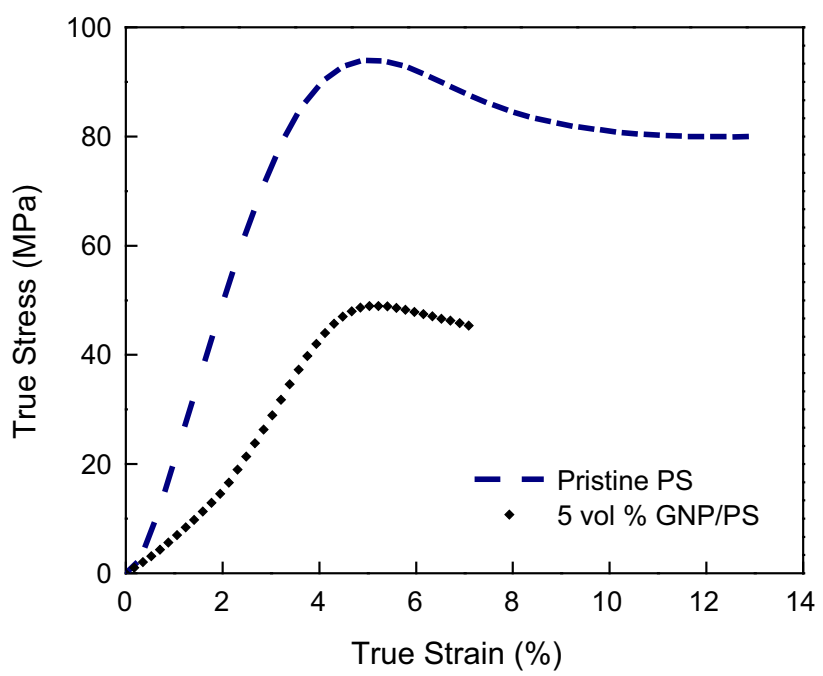

Fig. 10 True compressive stress-strain curve of pristine PS and GNP/PS under quasi-static loading
Fig. 9 SEM image of a crosssection of a post-mortem specimen loaded to $7 \%$ eng. strain

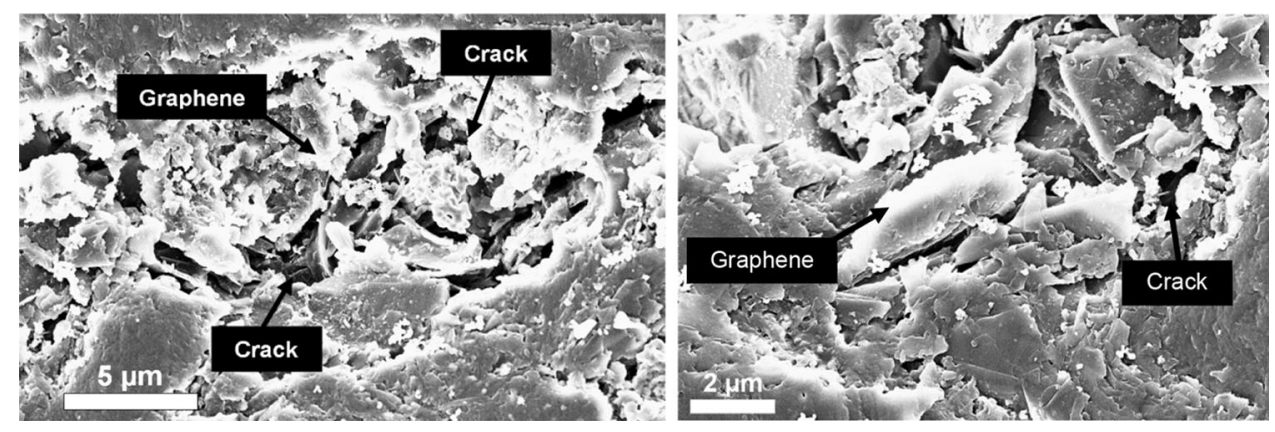




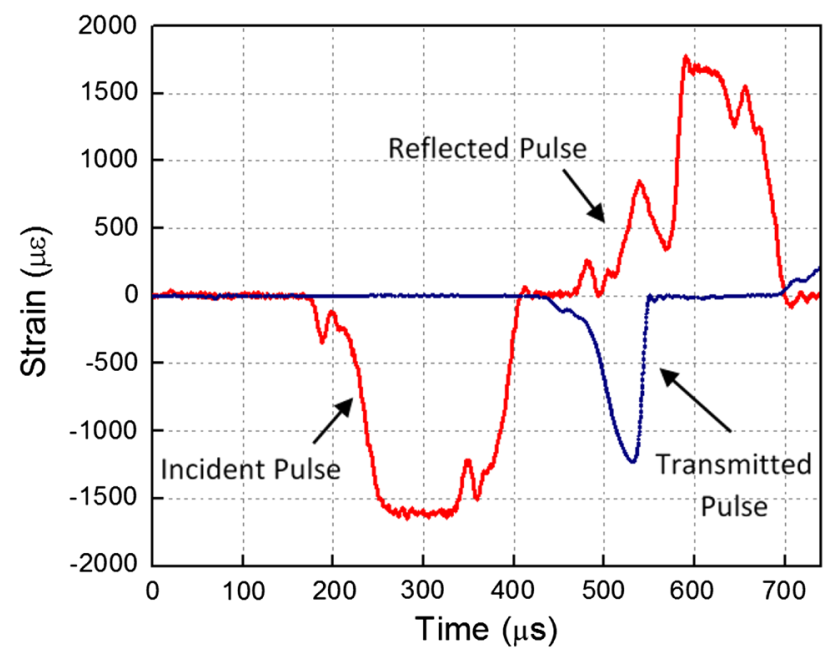

Fig. 11 Typical real-time strain pulses obtained from strain gages mounted on the bars for PS at an average strain rate of 2,000 s $\mathrm{s}^{-1}$

mechanical SHPB apparatus developed was utilized to load the specimens.

The real-time strain pulses obtained for PS at an average strain rate of $2,000 \mathrm{~s}^{-1}$ under dynamic compression are shown in Fig. 11. It can be observed from the figure that the pulse shaper, consisting of a layer of electrical tape and clay, used in all experiments helped to reduce high frequency oscillations in the incident stress wave. The incident pulse length applied to the specimen was $\sim 240 \mu \mathrm{s}$. Due to the brittle nature of the PS matrix, the transmitted pulse profile shows that the total loading event lasts only $\sim 100 \mu$ s at which point the specimen fails. It is important for the specimen to be in equilibrium under dynamic loading conditions for valid analysis of data. Figure 12 shows the typical force equilibrium of PS at an average strain rate of $2,000 \mathrm{~s}^{-1}$. The pulse shaper improved the force equilibrium conditions at the specimen-bar interfaces. The force equilibrium was maintained during the entire loading duration.

A typical electrical response along with the mechanical behavior of both neat PS and GNP reinforced composites is shown in Fig. 13. As the specimen undergoes dynamic compression, the electrical resistance increases proportional to the change in strain. As the stress of the specimen monotonically increases to $75 \mathrm{MPa}$ at $5 \%$ strain, the bulk electrical resistance of the specimen increases $\sim 85 \%$ due to the formation of micro-cracks within the matrix. As the internal damage grows, the electrical resistance continues to increase as the electrical efficiency of the composite is further diminished. The resistance does not abruptly jump but gradually increases as damage initiates and propagates throughout the composite. We believe that this difference may come from the non-uniform dispersion of graphene

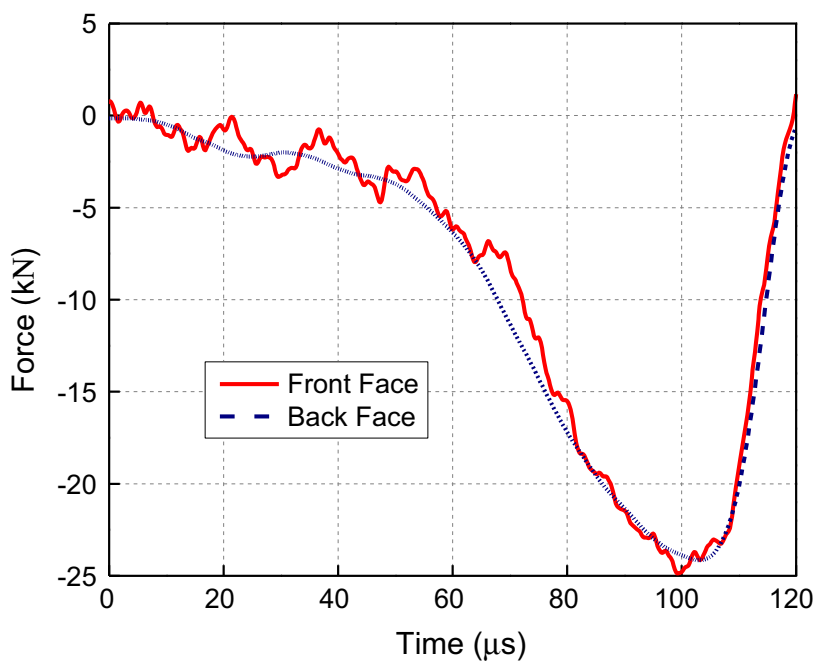

Fig. 12 Typical force equilibrium conditions at the specimen-bar interface at an average strain rate of $2,000 \mathrm{~s}^{-1}$

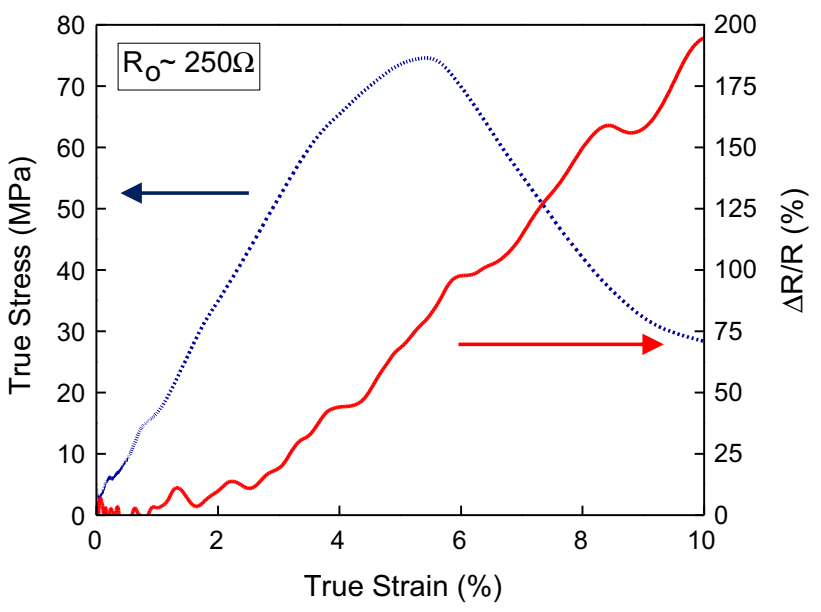

Fig. 13 Typical electro-mechanical response of 5 vol\% GNP/PS under dynamic loading

inside the matrix and complex initiation and propagation of damages.

A typical electrical response along with the real-time deformation images of a 5 vol\% GNP/PS composite subjected to dynamic loading are shown in Fig. 14. The time frames used in the loading event are chosen in a manner such that they can be correlated to the time at which certain deformation mechanisms were first observed. During the first $50 \mu \mathrm{s}$, the specimen undergoes a slight uniform compression. Since the strain of the material is minimal during this time, no noticeable change in electrical resistance is observed. At $\sim 60 \mu$ s, a surface crack is seen to initiate and propagate through the specimen consequently causing an increase in resistance. From 60 to $100 \mu$ s, damage further propagates throughout the specimen leading to larger increases in electrical resistance. 
Fig. 14 Percent change in electrical resistance of a $5 \mathrm{vol} \%$ GNP/PS composite subjected to SHPB loading with real-time deformation images

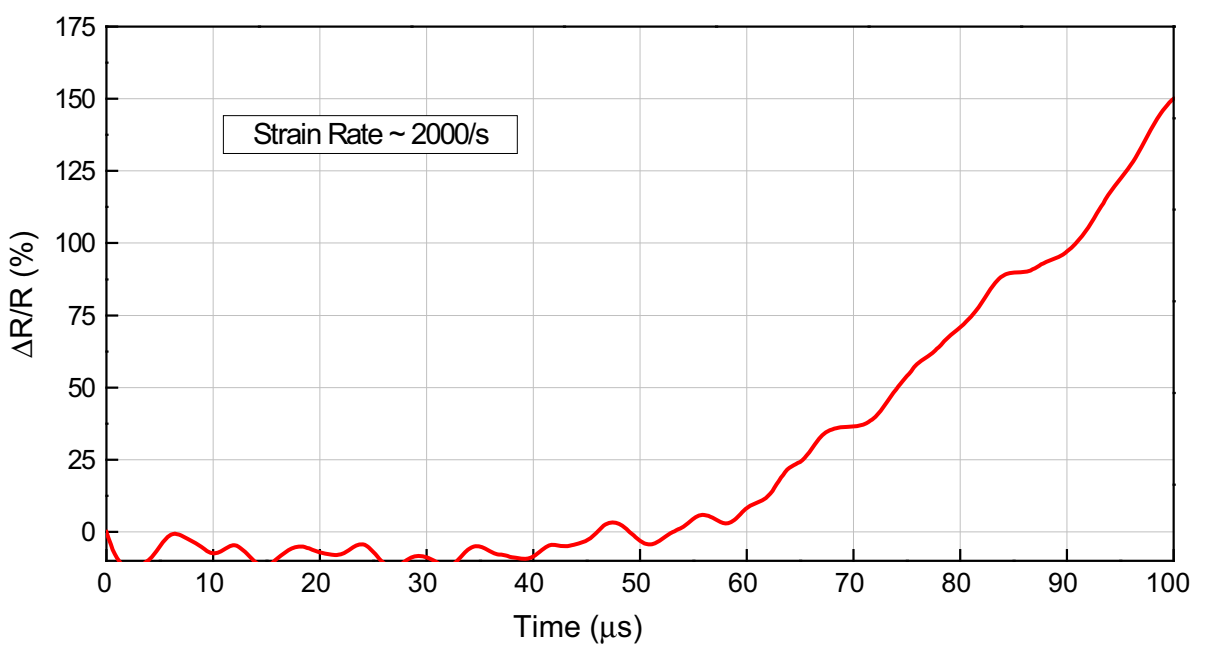

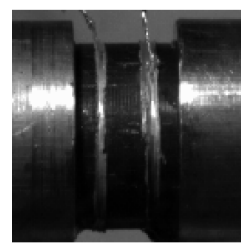

$0 \mu \mathrm{s}$

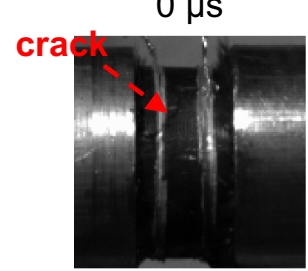

$60 \mu \mathrm{s}$

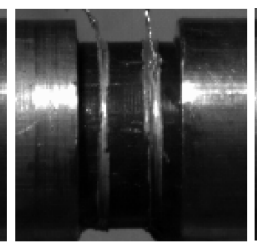

$20 \mu \mathrm{s}$

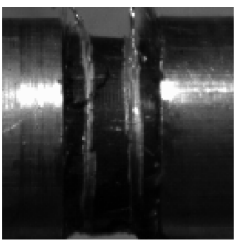

$70 \mu \mathrm{s}$

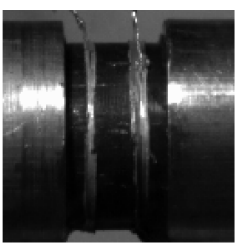

$30 \mu s$

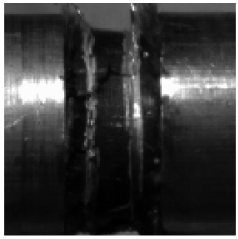

$80 \mu \mathrm{s}$

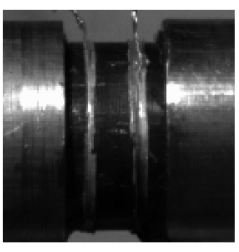

$40 \mu \mathrm{s}$

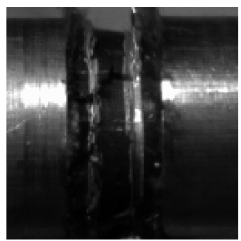

$90 \mu \mathrm{s}$

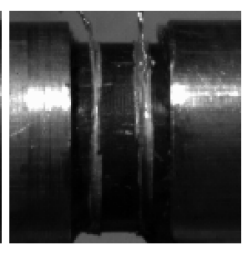

$50 \mu \mathrm{s}$

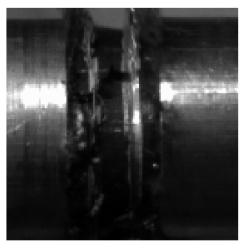

$100 \mu s$
The second series of dynamic experiments carried out were controlled-strain experiments in which the GNP/PS composites were dynamically loaded to a strain below the failure strain. Figure 15 shows the real-time dynamic electro-mechanical behavior of a 5 vol\% GNP/PS composite loaded to $5 \%$ strain. It can be observed that the resistance of the specimen begins to increase at $\sim 20 \mu \mathrm{s}$, while no significant change in compressive strain is observed until $\sim 100 \mu \mathrm{s}$. As previously discussed, the increase in resistance is a result of micro-crack formation within the composite due to the brittle nature of the PS matrix. As the strain increases to $\sim 1 \%$, the rate of change in resistance increases significantly due to the accumulating damage that continues to grow throughout the material. As the strain increases to $5 \%$, the specimen exhibits a $\sim 100 \%$ increase in resistance.

SEM imaging was performed on the post-mortem specimens to provide more insight on the internal damage mechanisms that occur during the dynamic loading. Figure 16 shows clear evidence of micro-crack formation through a cross-section of a 5 vol\% GNP/PS composite that

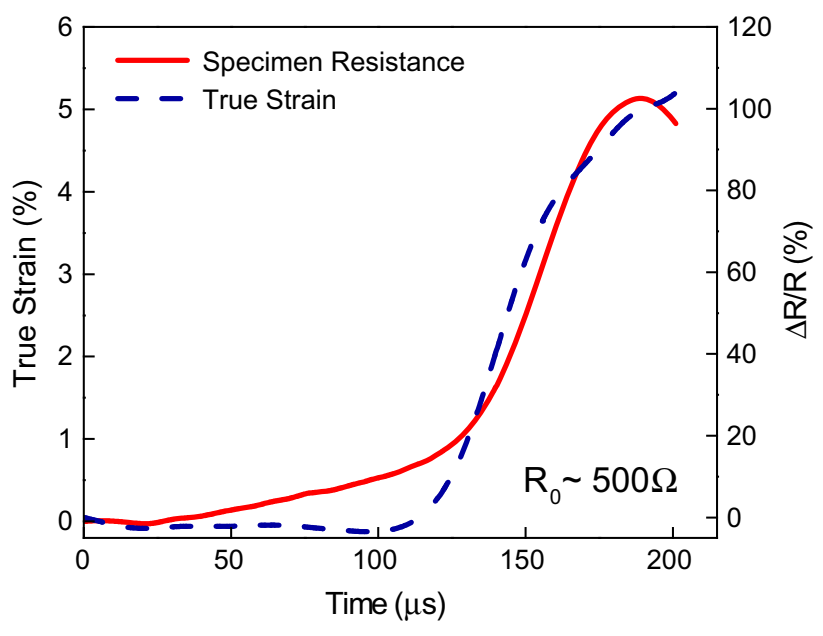

Fig. 15 Typical dynamic electro-mechanical behavior of a $5 \mathrm{vol} \%$ GNP/PS composite subjected to a controlled strain loading

was dynamically loaded to $5 \%$ strain. Similar to the damage mechanisms observed during quasi-static loading, it can be seen that the micro-cracks have formed primarily 
Fig. 16 SEM images of a cross-section of a post mortem specimen dynamically loaded to $5 \%$ true strain (arrows indicate GNPs)
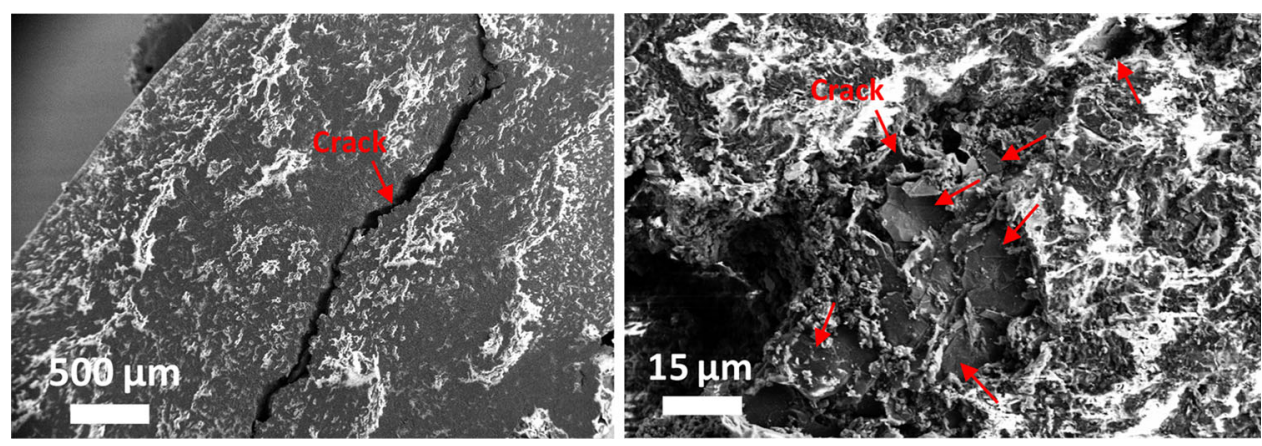

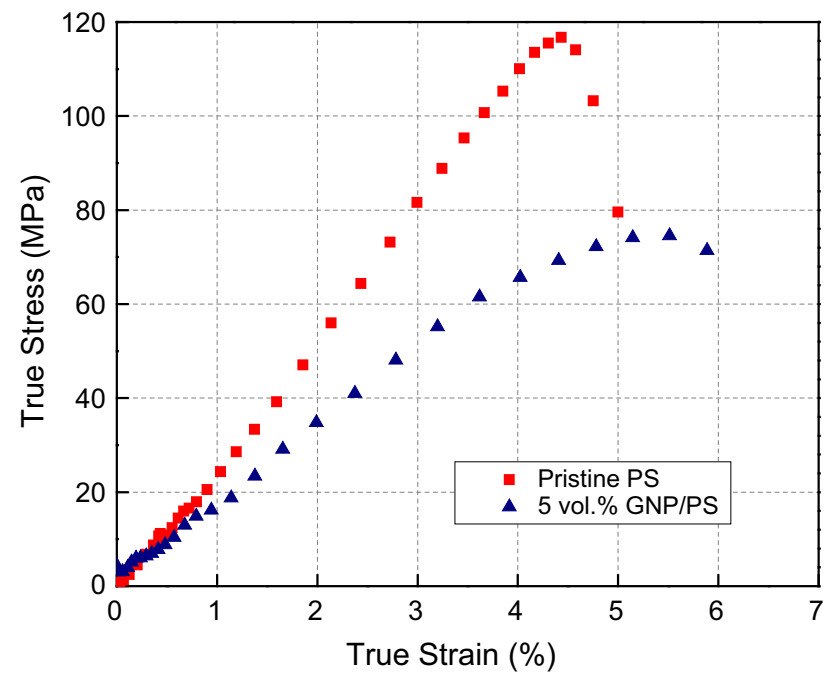

Fig. 17 True compressive stress-strain curve for pristine PS and $5 \mathrm{vol} \% \mathrm{GNP} / \mathrm{PS}$ at an average strain rate of $2,000 \mathrm{~s}^{-1}$

around small agglomerates of the GNPs. Again, these small agglomerates of the graphene sheets appear to serve as crack nucleation sites and due to poor interactions between the PS matrix and GNP particles, damage tends to propagate along the graphene-polymer interfaces.

The dynamic true stress-strain curves for pristine PS and 5 vol\% GNP/PS is shown in Fig. 17. The high strainrate yield stresses were much higher than the quasi-static ones for both pristine PS and GNP/PS composites. Similar to the static loading case, the GNP/PS composites demonstrated a reduced strength and modulus in comparison to pristine PS. The composite strength and modulus decreased $\sim 36$ and $\sim 66 \%$ respectively. Again, due to the high volume content of GNP, the reduced mechanical properties for this composite are attributed to the presence of relative agglomerations of GNPs within the PS matrix which prevent efficient load transfer to the graphene particles.

Figure 18 shows the effect of 5 vol\% GNP on the static and dynamic behavior of PS. Despite an increase in yield stress for dynamic loading in comparison to static loading, the presence of graphene within the PS matrix significantly

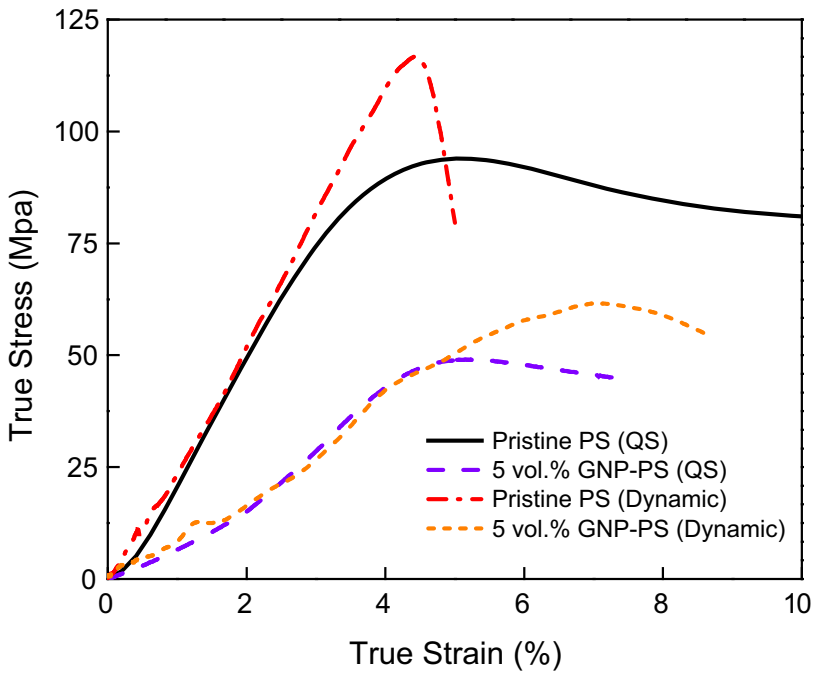

Fig. 18 Comparison of pristine PS versus GNP/PS under static and dynamic loading

diminishes the mechanical properties of the composite material under both static and dynamic compression.

The obtained results prove that the electro-mechanical SHPB can provide additional vital information that relates the transport properties to the evolution of the microstructure within these types of composites, even during the very early stages of loading, whereas a conventional SHPB cannot.

\section{Conclusions}

The present paper describes the electro-mechanical response of graphene reinforced PS composites under quasistatic and dynamic compressive loading. Graphene/PS composites with low electrical resistance were fabricated using a solution mixing approach followed by hot-pressing. A modified four-point probe method, using line and face contacts rather than point contacts, was implemented to accurately monitor the bulk electrical resistance of the composites. Moreover, a modified split Hopkinson (Kolsky) pressure bar apparatus, capable of simultaneous 
mechanical and electrical characterization, was developed and implemented to investigate the dynamic electro-mechanical response of the composites. In addition to measuring the change in electrical resistance as well as the dynamic constitutive behavior, real-time damage was captured using high-speed photography. The real-time damage was correlated to both stress-strain and percent change in resistance profiles. Due to a high concentration of graphene particles, relative aggregations of the graphene were inevitably formed which resulted in inadequate load transfer between the graphene particles and the PS matrix. Consequently, a significant decrease in mechanical properties under both static and dynamic loading conditions with the presence of graphene was observed. The bulk electrical resistance of the composite increased significantly due to the brittle nature of the PS matrix and the presence of relative agglomerations of graphene platelets which resulted in micro-crack formations. The obtained results showed that by implementing this type of electrical characterization technique, further insight regarding the evolution of micro-structure throughout these types of composites during both static and dynamic loading can be obtained.

Acknowledgments The authors acknowledge the financial support provided by the Rhode Island Science \& Technology Advisory Council as well as National Science Foundation (CMMI 1233887) Research Experiences for Undergraduates.

\section{References}

1. Frazier R, Daly D, Swatloski R, Hathcock K, South C (2009) Recent progress in graphene-related nanotechnologies. Recent Pat Nanotechnol 3:164-176

2. Godovsky DY (2000) Device applications of polymernanocomposites. In: Chang JY (ed) Biopolymers...PVA hydrogels, anionic polymerisation nanocomposites. Springer, Heidelberg, pp 163-205

3. Kim H, Miura Y, Macosko CW (2010) Graphene/polyurethane nanocomposites for improved gas barrier and electrical conductivity. Chem Mater 22(11):3441-3450

4. Kuilla T, Bhadra S, Yao D, Kim NH, Bose S, Lee JH (2010) Recent advances in graphene based polymer composites. Prog Polym Sci 35(11):1350-1375

5. Ramanathan T, Abdala AA, StankovichS Dikin DA, Herrera Alonso M, Piner RD et al (2008) Functionalized graphene sheets for polymer nanocomposites. Nat Nanotechnol 3(6):327-331

6. Soldano C, Mahmood A, Dujardin E (2010) Production, properties and potential of graphene. Carbon 48(8):2127-2150

7. Zhu Y, Murali S, Cai W, Li X, Suk JW, Potts JR et al (2010) Graphene and graphene oxide: synthesis, properties, and applications. Adv Mater 22(35):3906-3924

8. Cardoso SM, O'Connell CD, Pivonka R, Mooney C, Chalivendra VB, Shukla A, Yang SZ (2014) Effect of external loads on damage detection of rubber-toughened nanocomposites using carbon nanotubes sensory network. Polym Compos. doi:10.1002/ pc. 23188
9. Vadlamani VK, Chalivendra VB, Shukla A, Yang S (2012) Insitu sensing of non-linear deformation and damage in epoxy particulate composites. Smart Mater Struct. doi:10.1088/09641726/21/7/075011

10. Cardoso S, Chalivendra VB, Shukla A, Yang S (2012) Damage detection in the fracture process zone of rubber toughened epoxy using carbon nanotube sensory network. Eng Fract Mech 96:380-391

11. Vadlamani V, Chalivendra VB, Shukla A, Yang S (2012) Sensing of damage in carbon nanotubes and carbon black reinforced epoxy composites under tensile loading. Polym Compos 33(10):1809-1815

12. Singh V, Joung D, Zhai L, Das S, Khondaker SI, Seal S (2011) Graphene based materials: past, present and future. Prog Mater Sci 56(8):1178-1271

13. Fang M, Wang K, Lu H, Yang Y, Nutt S (2009) Covalent polymer functionalization of graphene nanosheets and mechanical properties of composites. J Mater Chem 19(38):7098-7105

14. Khan U, May P, O'Neill A, Coleman JN (2011) Development of stiff, strong, yet tough composites by the addition of solvent exfoliated graphene to polyurethane. Carbon 48(14):4035-4041

15. Liang J, Huang Y, Zhang L, Wang Y, Ma Y, Guo T et al (2009) Molecular-level dispersion of graphene into poly(vinyl alcohol) and effective reinforcement of their nanocomposites. Adv Funct Mater 19(14):2297-2302

16. Rath T, Li Y (2011) Nanocomposites based on polystyrene-bpoly(ethylene-r-butylene)-b-polystyrene and exfoliated graphite nanoplates: effect of nanoplatelet loading on morphology and mechanical properties. Compos A Appl Sci Manuf 42(12):1995-2002

17. Vadukumpully S, Paul J, Mahanta N, Valiyaveettil S (2011) Flexible conductive graphene/poly(vinyl chloride) composite thin films with high mechanical strength and thermal stability. Carbon 49(1):198-205

18. Yang S, Lin W, Huang Y, Tien H, Wang J, Ma C et al (2011) Synergetic effects of graphene platelets and carbon nanotubes on the mechanical and thermal properties of epoxy composites. Carbon 49(3):793-803

19. Goncalves G, Marques PAAP, Barros-Timmons A, Bdkin I, Singh MK, Emami N et al (2010) Graphene oxide modified with PMMA via ATRP as a reinforcement filler. J Mater Chem 20(44):9927-9934

20. Kim H, Macosko CW (2008) Morphology and properties of polyester/exfoliated graphite nanocomposites. Macromolecules 41(9):3317-3327

21. Yasmin A, Daniel IM (2004) Mechanical and thermal properties of graphite platelet/epoxy composites. Polymer 45(24):8211-8219

22. Sahrim H, Mou'ad A, Yahya S, Rozaidi R (2011) Reinforced thermoplastic natural rubber (TPNR) composites with different types of carbon nanotubes (MWNTS). In: Yellampalli S (ed) Carbon nanotubes-synthesis, characterization, applications. InTech, Chennai

23. Stankovich S, Dikin DA, Dommett GHB, Kohlhaas KM, Zimney EJ, Stach EA et al (2006) Graphene-based composite materials. Nature 442(7100):282-286

24. Wang L, Hong J, Chen G (2010) Comparison study of graphite nanosheets and carbon black as fillers for high density polyethylene. Polym Eng Sci 50(11):2176-2181

25. Kim H, Macosko CW (2009) Processing-property relationships of polycarbonate/graphene composites. Polymer 50(15):3797-3809

26. Qi X-Y, Yan D, Jiang Z, Cao Y-K, Yu Z-Z, Yavari F et al (2011) Enhanced electrical conductivity in polystyrene nanocomposites at ultra-low graphene content. ACS Appl Mater Interfaces 3(8):3130-3133

27. Kim I-H, Jeong YG (2010) Polylactide/exfoliated graphite nanocomposites with enhanced thermal stability, mechanical 
modulus, and electrical conductivity. J Polym Sci Part B: Polym Phys 48(8):850-858

28. Ramirez C, Figueiredo FM, Miranzo P, Poza P, Osendi MI (2012) Graphene nanoplatelet/silicon nitride composites with high electrical conductivity. Carbon 50(10):3607-3615

29. Zhang H-B, Zheng W-G, Yan Q, Yang Y, Wang J-W, Lu Z-H et al (2010) Electrically conductive polyethylene terephthalate/graphene nanocomposites prepared by melt compounding. Polymer 51(5):1191-1196

30. Heeder N, Shukla A, Chalivendra V, Yang S (2012) Sensitivity and dynamic electrical response of CNT-reinforced nanocomposites. J Mater Sci 47(8):3808-3816

31. Heeder NJ, Shukla A, Chalivendra V, Yang S, Park K (2012) Electrical response of carbon nanotube reinforced nanocomposites under static and dynamic loading. Exp Mech 52(3):315-322

32. Lim AS, An Q, Chou T-W, Thostenson ET (2011) Mechanical and electrical response of carbon nanotube-based fabric composites to Hopkinson bar loading. Compos Sci Technol 71(5):616-621

33. Thostenson ET, Chou TW (2006) Carbon nanotube networks: sensing of distributed strain and damage for life prediction and self healing. Adv Mater 18(21):2837-2841
34. Eswaraiah V, Balasubramaniam K, Ramaprabhu S (2011) Functionalized graphene reinforced thermoplastic nanocomposites as strain sensors in structural health monitoring. J Mater Chem 21(34):12626-12628

35. Li W, Dichiara A, Bai J (2013) Carbon nanotube-graphene nanoplatelet hybrids as high-performance multifunctional reinforcements in epoxy composites. Compos Sci Technol 74:221-227

36. Qu S, Wong S-C (2007) Piezoresistive behavior of polymer reinforced by expanded graphite. Compos Sci Technol 67(2):231237

37. Bianco A, Cheng H-M, Enoki T, Gogotsi Y, Hurt RH, Koratkar N et al (2013) All in the graphene family-a recommended nomenclature for two-dimensional carbon materials. Carbon 65:1-6

38. Kolsky H (1949) An investigation of the mechanical properties of materials at very high rates of loading. Proc Phys Sci 62:676-700

39. Song B, Chen W (2004) Loading and unloading split Hopkinson pressure bar pulse-shaping techniques for dynamic hysteretic loops. Exp Mech 44(6):622-627

40. Tjong SC (2006) Structural and mechanical properties of polymer nanocomposites. Mater Sci Eng R: Rep 53(3-4):73-197 